\title{
BIOCHEMICAL AND PHARMACOLOGICAL STUDY OF VENOM OF THE WOLF SPIDER Lycosa singoriensis
}

\author{
Liu ZH (1), Qian W (2), Li J (1), Zhang Y (1), Liang S (1)
}

(1) College of Life Sciences, Hunan Normal University, Changsha, China; (2) Administrative Center for Basic Research, Ministry of Science ant Technology, China.

ABSTRACT: The wolf spider Lycosa singoriensis is a large and venomous spider distributed throughout northwestern China. Like other spider venoms, the wolf spider venom is a chemical cocktail. Its protein content is $0.659 \mathrm{mg}$ protein $/ \mathrm{mg}$ crude venom as determined by the Lowry method. MALDI-TOF analysis revealed that the venom peptides are highly diverse and may be divided into three groups characterized by three independent molecular ranges: 2,000 to $2,500 \mathrm{Da}, 4,800$ to $5,500 \mathrm{Da}$ and 7,000 to $8,000 \mathrm{Da}$, respectively. This molecular distribution differs substantially from those of most spider venoms studied so far. This wolf spider venom has low neurotoxic action on mice, but it can induce hemolysis of human erythrocytes. Furthermore, the venom shows antimicrobial activity against prokaryotic and eukaryotic cells.

KEY WORDS: spider, Lycosa singoriensis, crude venom, MALDI-TOF, antimicrobial activity.

CONFLICTS OF INTEREST: There is no conflict.

FINANCIAL SOURCE: National Science Foundation, China.

\section{CORRESPONDENCE TO:}

SONGPING LIANG, College of Life Sciences, Hunan Normal University, Changsha, Hunan, 410081, China. Phone: +86 731 8872556. Fax: +86 731 8861304. Email: liangsp@hunnu.edu.cn. 


\section{INTRODUCTION}

There are about 39,000 described spider species, with an even greater number awaiting characterization. Almost all spiders are predators and have venom glands. The primary propose of spider venoms is to kill or paralyze prey. Spider venoms are complex chemical cocktails in which peptides are the principal constituents of most spider venoms, except those of black widow spiders that contain a high proportion, greater than $100 \mathrm{kD}$ proteins (1-3). The spider venom peptides are produced in a combinatorial fashion, which leads to an estimated total of about 1.5 million spider venom peptides. Consequently, spider venoms are a rich source of pharmacologically and agrochemically interesting novel compounds that have received increased attention from pharmacologists and biochemists in recent years. However, during the past decades, only a few spider venoms have been studied in sufficient detail, and therefore less than $0.01 \%$ of spider venom peptides have been identified thus far (4-7). The wolf spider Lycosa singoriensis is a large spider distributed throughout northwest China. The adult female spider has a body length from 28 to $40 \mathrm{~mm}(35 \pm 6 \mathrm{~mm})$ and a body weight from 2.6 to $7 \mathrm{~g}$ (Figure 1). This hairy spider lives in holes underground. Its burrow, lined with a silk tube, has a diameter from 2 to $4 \mathrm{~cm}$ and a length from 30 to 60 $\mathrm{cm}$, and the burrow entrance is often covered with silk net. The spider spends the day huddled at the bottom of the hole, while it ascends the silk tube and hides itself near the burrow entrance waiting for prey at night. After succeeding in catching victims, the spider brings them into the hole. In many cases, residues of small insects are found at the bottom of the burrow. The wolf spider Lycosa singoriensis is also a venomous and aggressive spider. In 2000, it was reported that there were wolf spider bites to humans and other animals in the northern area of Xinjiang province. According to clinical records, the majority of the spider bites caused apparent effects, including red marks and pain around the bite sites $(8,9)$.

In this study, we report the biochemical and pharmacological properties of the venom of the wolf spider Lycosa singoriensis. Compared with many other spider venoms studied so far, this spider venom has some distinct properties, which makes it a useful source for screening drug leads and for studying biodiversity of spider venom peptides. 


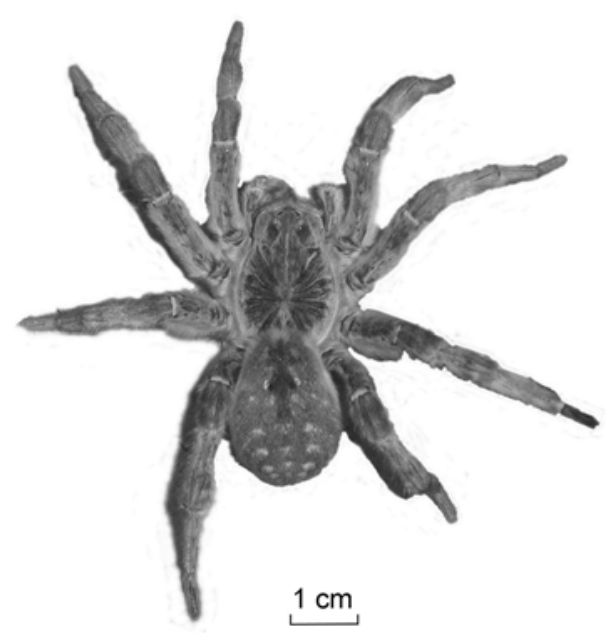

Figure 1. A female wolf spider Lycosa singoriensis. This hairy spider has a body length from 28 to $40 \mathrm{~mm}$.

\section{MATERIALS AND METHODS}

\section{Spiders and Venom Collection}

Adult female Lycosa singoriensis spiders were collected in Xinjiang province, China, maintained in plastic buckets that were covered with plastic nets and given water daily. Chopped pig livers and worms were used to feed the animals. Like many other large spiders $(10,7)$, Lycosa singoriensis readily become aggressive when provoked by a piece of plastic tubing. They grasp the tubing tightly, and then its venom fangs pierce the tubing and inject venom inside. This avoids the need for electrical stimulation, which can contaminate venom with enzymes from both saliva and digestive fluids. Using this method could yield about $50 \mathrm{mg}$ of venom from approximately 300 Lycosa singoriensis spiders, thus enabling exploration of the biochemical and pharmacological properties of this spider venom. The crude venom is a clear and colorless liquid, easily soluble in water and was collected every two weeks. Freeze-dried crude venom was stored at $-20^{\circ} \mathrm{C}$ prior to analysis.

\section{SDS-PAGE Analysis of Crude Venom}

Sodium dodecyl sulfate polyacrylamide gel electrophoresis (SDS-PAGE) on the 
collected venom was performed under denatured conditions in a $10 \%$ polyacrylamide slab gel. One hundred micrograms of lyophilized venom was used for electrophoresis, and the separated proteins in the gel were visualized by G250 staining.

\section{MALDI-TOF Analysis of Crude Venom}

The crude venom fingerprint was determined using a matrix-assisted laser desorption/ionization-time-of-flight (MALDI-TOF) mass spectrometry (Voyager-DE STR Biospectometry ${ }^{\circledR}$ workstation, Applied Biosystems, USA). Ionization was achieved by irradiation with a nitrogen laser $(337 \mathrm{~nm})$ at a $20-\mathrm{kV}$ acceleration voltage; a-cyano-4-hydroxy-cinnamic acid (CCA) was employed as matrix.

\section{The Effect of Crude Venom on Isolated Nerve-Synapse Preparations}

Three types of isolated nerve-synapse preparations - mouse phrenic nerve-diaphragm, rat vas deferens and toad heart - were used to investigate the pharmacological activity of the crude venom. The mouse phrenic nerve-diaphragm preparation experiments were carried out according to Bülbring (11). Vas deferens assays and toad heart assays were performed according to Liang et al. (12).

\section{Hemolytic Assay}

Hemolytic activity of the crude venom was assayed with heparinized human red blood cells rinsed three times in $5 \mathrm{~mL}$ phosphate buffered saline (PBS - $50 \mathrm{mM} \mathrm{NaH}_{2} \mathrm{PO}_{4}$ and $150 \mathrm{mM} \mathrm{NaCl}, \mathrm{pH}$ 7.2) and centrifuged for 5 minutes at 3,000 rpm. Red blood cells were then incubated at room temperature for 1 hour in deionized water (positive control), in PBS (blank), or with venoms at various concentrations (3.1 to $20 \mathrm{mg} / \mathrm{mL}$ ) in PBS. The samples were centrifuged at 12,000 rpm for 5 minutes. The supernatant was separated from the pellet, and its absorbance was measured at $570 \mathrm{~nm}$.

\section{Antimicrobial Activity of Crude Venom}

Six bacteria (Bacillus cereus, Corynebacterium glutamicum, Bacillus subtilis, 
Micrococcus luteus, Staphylococcus albus and E. coli DH5) and two fungi (Saccaromyces cerevisae and Candida albicans) were respectively grown in the culture medium until reaching exponential phase with an absorbance at $600 \mathrm{~nm}$ of 0.3 to 0.8 . Fifty microliters of medium was spread uniformly on three solidified agar plates. The plates were completed with $1.5 \%$ agarose/medium poured into $100 \times 20 \mathrm{~mm}$ sterile Petri dishes. A 6-mm-diameter filter paper covered the plates. Five microliters of venom solution, in normal saline, at various concentrations was placed onto the filter paper. After incubation at $37^{\circ} \mathrm{C}$ overnight, the effects of the crude venom were recorded as clear circles in the bacterial lawn on the filter paper.

Therefore, in this bioassay, crude venom solutions at various concentrations $(3 \mathrm{mg} / \mathrm{mL}$, $6 \mathrm{mg} / \mathrm{mL}$ and $12 \mathrm{mg} / \mathrm{mL}$ ) were dropped onto the filter paper, and a clear circle was detectable on the paper if the venom at this concentration had inhibited the microbial growth.

\section{RESULTS AND DISCUSSION}

\section{Biochemical Characterization of Crude Venom}

It was found that each milligram of crude venom contains about $0.659 \mathrm{mg}$ protein/peptides. As displayed in Figure 2, the high-molecular-mass proteins of the crude venom are distributed mainly among molecular masses ranging from 14 to 31,000 Da, with a thick protein band near 20,000 Da and another band evident near $14,000 \mathrm{Da}$. A thick band is also visible on the top of the panel of the SDS-PAGE gel which consists of the peptides with molecular masses less than 10,000 Da. The proteins/peptides distributed in the two thick bonds are the most abundant components of the crude venom, which correspond to $80 \%$ of the protein component of the crude venom. 


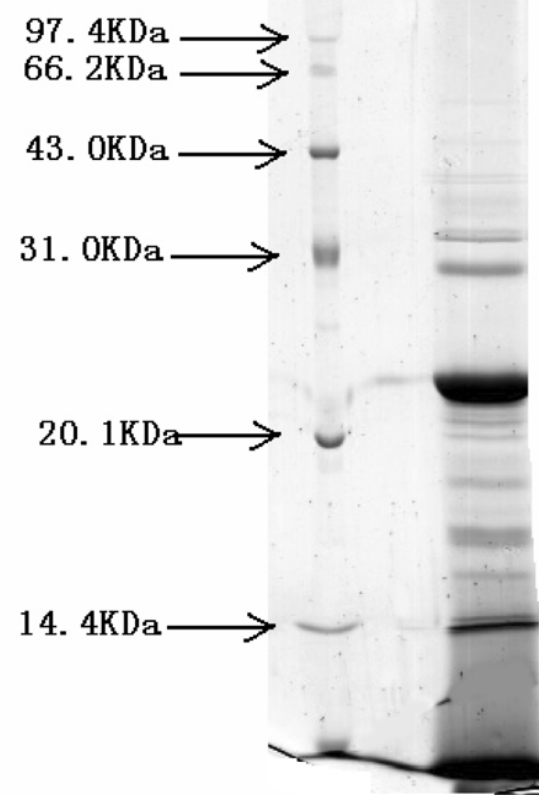

Figure 2. SDS-PAGE image of Lycosa singoriensis crude venom.

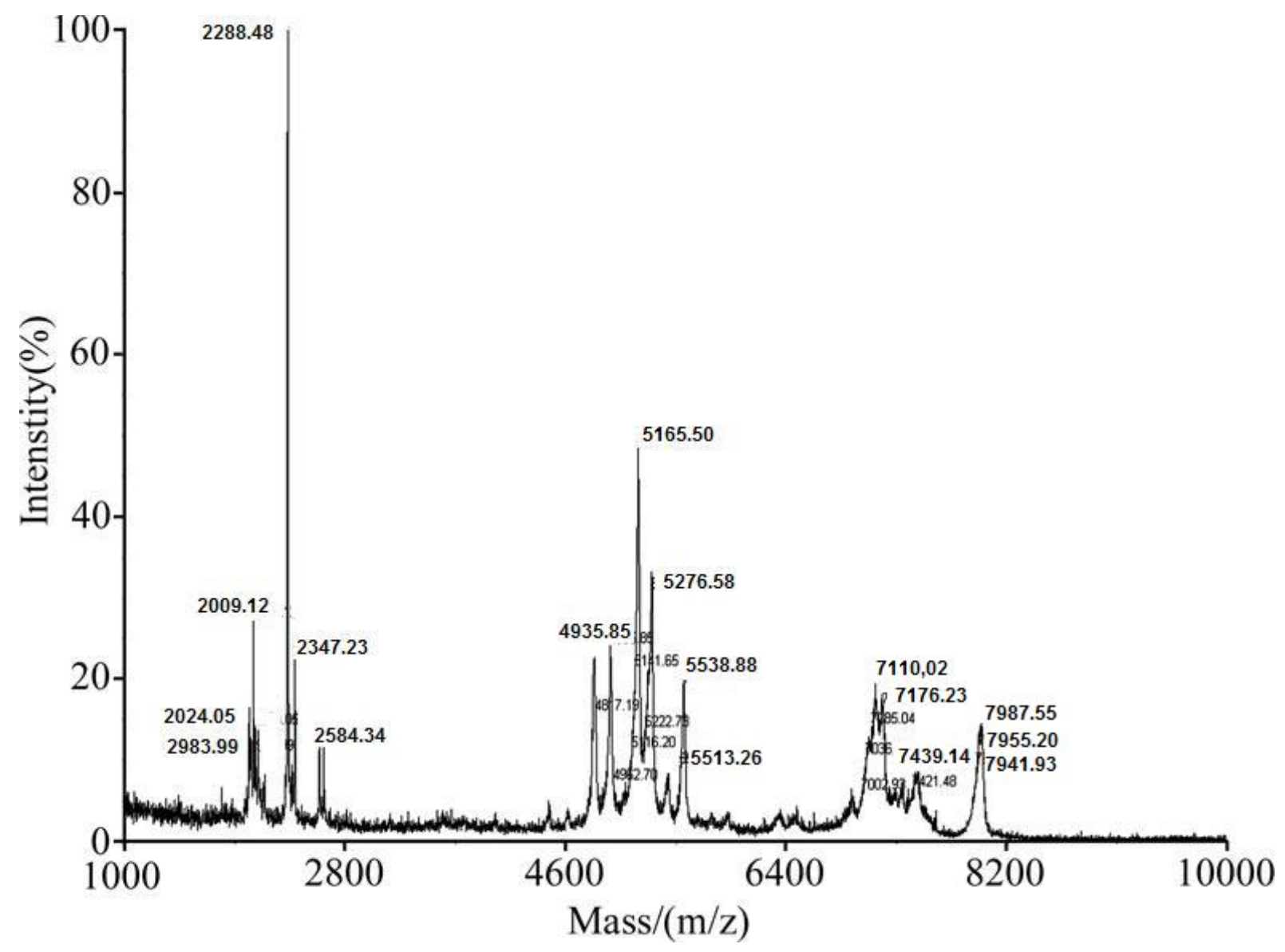

Figure 3. MALDI-TOF spectrum of Lycosa singoriensis crude venom. 
Recently, MALDI-TOF mass spectrometry was applied to elucidate the complexity of venom peptides. Additionally, with the rapid development of mass spectrometry, this technology has been widely used in venom research $(5,13)$. For example, Pierre Escoubas et al. (5) painted a venom picture to conceptualize the complexity of Australian funnel-web spider venoms by using a combined cDNA and mass spectrometric approach. Their studies demonstrate that the venoms from these spiders contain many hundreds of peptides that follow a bimodal distribution, with the majority of peptides in the 3,000-to-5,000 Da mass range, and a second less pronounced group in the 6,500-to-8,500 Da range. This distribution of molecular masses is analogous to that observed previously for a large number of tarantula venoms (4). Similar results were also found in our previous studies of venoms from the Chinese tarantula spiders Ornithoctonus huwena, Ornithoctonus hainana and Chilobrachys jingzhao (14-16).

As presented in Figure 3, the MALDI-TOF spectrometry analysis of Lycosa singoriensis crude venom demonstrates that the molecular mass distribution is analogously observed in the range from 1,000 to $10,000 \mathrm{Da}$. However, unlike those aforementioned species, Lycosa singoriensis venom peptides can be divided into three groups by their masses. The first group comprises peptides that possess molecular masses between 2,000 and 2,500 Da, which indicates that they have about 20 amino acid residues. This molecular mass range has rarely been observed in most spider venoms studied to date. The second group includes mainly peptides with molecular masses in the 4,800-to-5,500-Da range, suggesting that they are composed of about 50 amino acid residues. The third group consists of peptides with mass range from 7,000 to $8,000 \mathrm{Da}$, which corresponds to more than 60 amino acid residues. It is estimated that peptides distributed in the latter two groups are the majority of the venom peptides.

Interestingly, rather few peptides have molecular masses between 3,000 and 5,000 Da. Paradoxically, peptides in this mass range are the most abundant component in many other spider venoms. Further studies would be needed to elucidate these discrepancies, which may contribute to understanding the evolutionary mechanism of 
spider venom peptides. The current study contributes to proving that Lycosa singoriensis peptides are highly diverse.

Analysis of venom-gland cDNA libraries yielded more than 200 toxin-like peptide sequences. The mass distribution of these peptides derived from cDNA sequences is consistent with that observed by MALDI-TOF spectrometry. Furthermore, analysis of the sequences revealed that peptides from the first group have no cysteine residues, and the ones of the second group contain 4 or 5 disulfide bonds, whereas those of the last group have more than 5 disulfide bonds (unpublished data). Most spider peptide toxins identified until now usually have 3 or 4 disulfide bonds, and their 3D structures adopt the classical inhibitor cystine-knot motif. Consequently, there is reason to believe that some peptides from Lycosa singoriensis venom would possess novel structural theme.

\section{Pharmacological Characterization of Crude Venom}

The crude venom, at a high dose of $200 \mu \mathrm{g} / \mathrm{mL}$, could not block the electrically-stimulated contraction of mouse phrenic nerve diaphragm preparation $(n=$ 5). It also exhibited low effect on the twitch response of rat vas deferens. The concentration of $200 \mu \mathrm{g} / \mathrm{mL}$ of crude venom could only partially inhibit the twitch response for 20 minutes $(n=5)$ (Figure $4-A)$. In contrast, the crude venom of $O$. huwena spider, at the same concentration, was able to rapidly block the twitch response of the same nerve-diaphragm preparation or rat vas deferens (data not shown). However, Lycosa singoriensis crude venom had significant effect on toad heart contraction. In the presence of $100 \mu \mathrm{g} / \mathrm{mL}$ of crude venom, the rate and magnitude of the heartbeat were potently augmented $(n=5)$ (Figure $4-B)$. This suggests that the crude venom contains some compounds that are cardiotonic.

Currently, cardiotonic agents are classified into three classes based on their subcellular action mechanisms, namely, agents acting through upstream mechanisms $\left(\mathrm{Ca}^{2+}\right.$ mobilizers), as well as central and downstream mechanisms $\left(\mathrm{Ca}^{2+}\right.$ sensitizers). These agents induce a positive inotropic effect by elevating the intracellular $\mathrm{Ca}^{2+}$ ion concentration (17). To date, there is no report of a cardiotonic effect of wolf spider 
venoms while no cardiotonic compounds have yet been purified and characterized from these venoms. Therefore, it is important to investigate the cardiotonic compounds from Lycosa singoriensis venom.

A

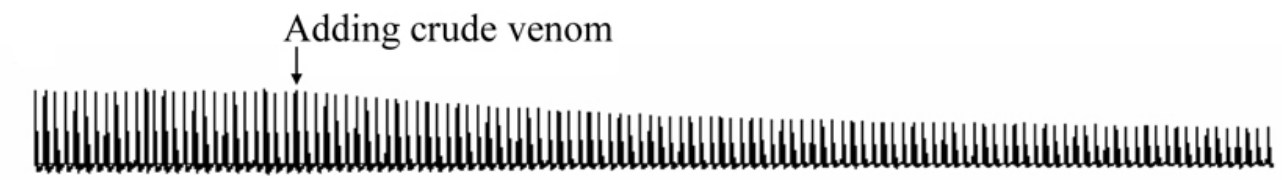

B
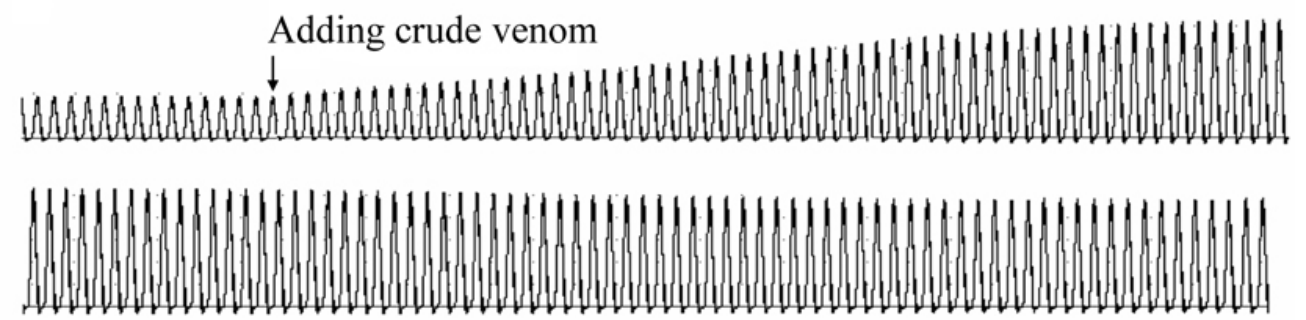

Figure 4. The effects of the crude venom of Lycosa singoriensis on the contraction of rat vas deferens $(\mathbf{A})$ and toad heart (B).

The hemolytic activity of the crude venom was determined by using fresh human erythrocytes. As shown in Figure 5 (A), the crude venom disrupted human erythrocytes in a dose-dependent manner. Its effective concentration of $50 \%$ inhibition $\left(E_{50}\right)$ value is $1.25 \mathrm{mg} / \mathrm{mL}$.

As reported by Budnik et al. (18), Lycosa singoriensis crude venom contains antimicrobial peptides (denominated lycocitins 1,2 and 3) that can inhibit the growth of gram-positive and gram-negative bacteria and fungi at micromolar concentrations. We, therefore, tested the antimicrobial activity of the crude venom against both prokaryotic and eukaryotic cells by the plate growth inhibition assay. Under our bioassay conditions, the cell strains most sensitive to the crude venom were, specifically, Bacillus subtilis and Staphylococcus sp, in which the growth was potently inhibited at 3 $\mathrm{mg} / \mathrm{mL}$. The venom also acted strongly against Corynebacterium glutamicum and Micrococcus luteus but weakly against one of the fungi strains (Candida albicans). However, the crude venom had no detectable effect on E. coli and Saccaromyces cerevisae even at the high concentration of $12 \mathrm{mg} / \mathrm{mL}$ (Figure $5-\mathrm{B}$ ). 
$(\mathrm{mg} / \mathrm{mL})$

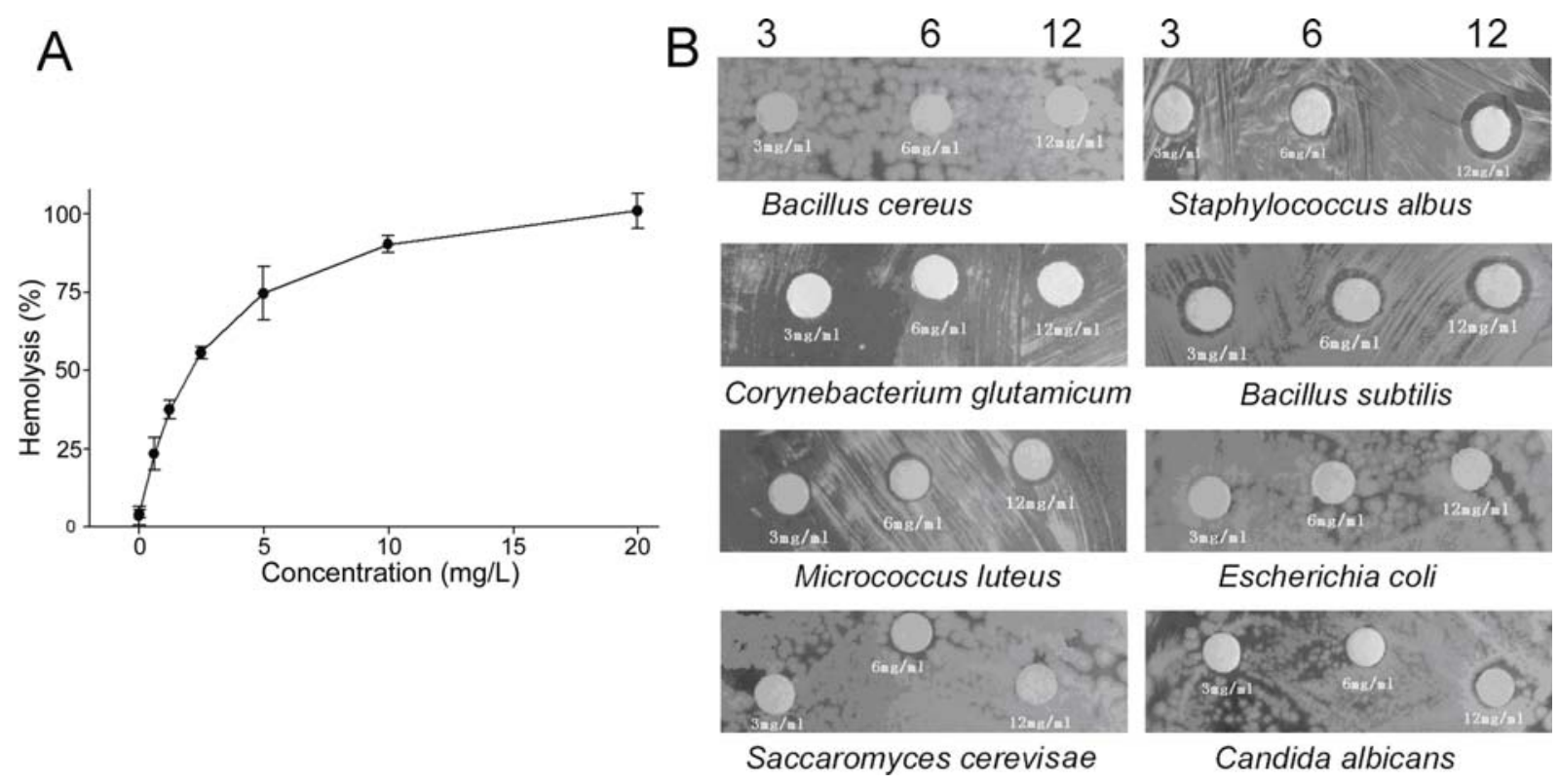

Figure 5. The cytotoxic activity of Lycosa singoriensis crude venom. The crude venom has hemolytic activity against human erythrocytes with an $\mathrm{EC}_{50}$ value of $1.25 \mathrm{mg} / \mathrm{mL}$ (A). The crude venom has antimicrobial activity against both prokaryotic and eukaryotic cells in the plate growth inhibition assay (B). The numbers on the top of the panel show the crude venom concentrations in $\mathrm{mg} / \mathrm{mL}$.

During the past ten years, many antimicrobial peptides have been identified from spider venoms. Lycotoxins I and II were identified from the venom of the wolf spider Lycosa carolinensis. Both are linear antimicrobial peptides that demonstrate the amphipathic $\alpha$-helix character typical of pore-forming peptides. Their pore-forming mechanism was further verified by their promoting efflux of calcium ions from synaptosomes (19). Subsequent to the lycotoxins, cupiennins (20-22) and oxyopinins $(23,24)$, from the venom of the wolf spiders Cupiennus salei and Oxyopes kitabensis, respectively, were also found to have antimicrobial activities. More recently, seven novel short linear antimicrobial and cytolytic peptides called latarcins were purified from the venom of Lachesana tarabaevi spider. In addition, five novel peptides that share considerable structural similarity with the purified latarcins were predicted from expressed sequence tag data base for the spider venom gland (25).

These peptides from spider venoms belong to linear cationic $\alpha$-helical antimicrobial 
peptides. This class of antimicrobial peptides shares some common characteristics, such as inhibiting microbial growth at low micromolar concentrations and forming amphipathic and cationic helical formation in hydrophobic environments. During the recent decades, a large number of antimicrobial peptides, including linear cationic a-helical antimicrobial peptides, have been discovered in animals as well as plants. Those peptides are typically composed of 12 to 45 amino acids, and play important roles in the innate immune systems of most living organisms (26-28). Most of them can kill microorganisms with the following four features: selective toxicity, rapid killing, broad antimicrobial spectra, and no resistance development (29-31).

In summary, we report new biochemical and pharmacological findings on the venom of wolf spider Lycosa singoriensis. The distinct properties of the peptides of this venom make it an ideal model to study the evolutionary mechanisms of spider venom peptides. The pharmacological study of this venom is helpful for purifying and characterizing bioactive peptides in further studies.

\section{ACKNOWLEDGMENTS}

This work was supported by National Science Foundation Projects (30430170 and 30700127).

\section{REFERENCES}

1. Corzo G, Escoubas P. Pharmacologically active spider peptide toxins. Cell Mol Life Sci. 2003;60(11):2409-26.

2. Escoubas P. Molecular diversification in spider venoms: a web of combinatorial peptide libraries. Mol Divers. 2006;10(4):545-54.

3. King GF. The wonderful world of spiders: preface to the special Toxicon issue on spider venoms. Toxicon. 2004;43(5):471-5.

4. Escoubas P, Rash L. Tarantulas: eight-legged pharmacists and combinatorial chemists. Toxicon. 2004;43(5):555-74. 
5. Escoubas P, Sollod B, King GF. Venom landscapes: mining the complexity of spider venoms via a combined cDNA and mass spectrometric approach. Toxicon. 2006;47(6):650-63.

6. Sollod BL, Wilson D, Zhaxybayeva O, Gogarten JP, Drinkwater R, King GF. Were arachnids the first to use combinatorial peptide libraries? Peptides. 2005;26(1):131-9. 7. Tedford HW, Sollod BL, Maggio F, King GF. Australian funnel-web spiders: master insecticide chemists. Toxicon. 2004;43(5):601-18.

8. Lu DL, Zhang DF. Two kinds of poisonous spiders in Xinjiang and prevention and cure for the spider bite. Chin J Zool. 2001;36(5):40-2.

8. Initial observation on living habit of Lycosa singorensis. Chin J Zool. 2004;39:63-7. 10. Liu Z, Dai J, Dai L, Deng M, Hu Z, Hu W, Liang S. Function and solution structure of huwentoxin- $\mathrm{X}$, a specific blocker of $\mathrm{N}$-type calcium channels, from the Chinese bird spider Ornithoctonus huwena. J Biol Chem. 2006;281(13):8628-35.

11. Bulbring E. Observations on the isolated phrenic nerve diaphragm preparation of the rat. Br J Pharmacol Chemother. 1946;1(1):38-61.

12. Liang SP, Chen XD, Shu Q, Zhang Y, Peng K. The presynaptic activity of huwentoxin-I, a neurotoxin from the venom of the Chinese bird spider Selenocosmia huwena. Toxicon. 2000;38(9):1237-46.

13. Guette C, Legros C, Tournois G, Goyffon M, Célérier ML. Peptide profiling by matrix-assisted laser desorption/ionisation time-of-flight mass spectrometry of the Lasiodora parahybana tarantula venom gland. Toxicon. 2006;47(6):640-9.

14. Liang S. An overview of peptide toxins from the venom of the Chinese bird spider Selenocosmia huwena Wang [=Ornithoctonus huwena (Wang)]. Toxicon. 2004;43(5):575-85.

15. Liao Z, Cao J, Li S, Yan X, Hu W, He Q, Chen J, Tang J, Xie J, Liang S. Proteomic and peptidomic analysis of the venom from Chinese tarantula Chilobrachys jingzhao. Proteomics. 2007;7(11):1892-907.

16. Yuan C, Jin Q, Tang X, Hu W, Cao R, Yang S, Xiong J, Xie C, Xie J, Liang S. Proteomic and peptidomic characterization of the venom from the Chinese bird spider, Ornithoctonus huwena Wang. J Proteome Res. 2007;6(7):2792-801. 
17. Endoh M. A $\mathrm{Na}^{+}$channel agonist: a potential cardiotonic agent with a novel mechanism? Br J Pharmacol. 2004;143(6):663-5.

18. Budnik BA, Olsen JV, Egorov TA, Anisimova VE, Galkina TG, Musolyamov AK, Grishin EV, Zubarev RA. De novo sequencing of antimicrobial peptides isolated from the venom glands of the wolf spider Lycosa singoriensis. J Mass Spectrom. 2004;39(2):193-201.

19. Yan L, Adams ME. Lycotoxins, antimicrobial peptides from venom of the wolf spider Lycosa carolinensis. J Biol Chem. 1998;273(4):2059-66.

20. Kuhn-Nentwig L, Dathe M, Walz A, Schaller J, Nentwig W. Cupiennin 1d: the cytolytic activity depends on the hydrophobic $\mathrm{N}$-terminus and is modulated by the polar C-terminus. FEBS Lett. 2002;527(1-3):193-8.

21. Kuhn-Nentwig L, Muller J, Schaller J, Walz A, Dathe M, Nentwig W. Cupiennin 1, a new family of highly basic antimicrobial peptides in the venom of the spider Cupiennius salei (Ctenidae). J Biol Chem. 2002;277(13):11208-16.

22. Pukala TL, Doyle JR, Llewellyn LE, Kuhn-Nentwig L, Apponyi MA, Separovic F, Bowie $\mathrm{JH}$. Cupiennin 1a, an antimicrobial peptide from the venom of the neotropical wandering spider Cupiennius salei, also inhibits the formation of nitric oxide by neuronal nitric oxide synthase. FEBS J. 2007;274(7):1778-84.

23. Corzo G, Villegas E, Gomez-Lagunas F, Possani LD, Belokoneva OS, Nakajima T. Oxyopinins, large amphipathic peptides isolated from the venom of the wolf spider Oxyopes kitabensis with cytolytic properties and positive insecticidal cooperativity with spider neurotoxins. J Biol Chem. 2002;277(26):23627-37.

24. Nomura K, Corzo G. The effect of binding of spider-derived antimicrobial peptides, oxyopinins, on lipid membranes. Biochim Biophys Acta. 2006;1758(9):1475-82.

25. Kozlov SA, Vassilevski AA, Feofanov AV, Surovoy AY, Karpunin DV, Grishin EV. Latarcins, antimicrobial and cytolytic peptides from the venom of the spider Lachesana tarabaevi (Zodariidae) that exemplify biomolecular diversity. J Biol Chem. 2006;281(30):20983-92.

26. Brogden KA. Antimicrobial peptides: pore formers or metabolic inhibitors in bacteria? Nat Rev Microbiol. 2005;3(3):238-50. 
27. Brown KL, Hancock RE. Cationic host defense (antimicrobial) peptides. Curr Opin Immunol. 2006;18(1):24-30.

28. Zasloff M. Antimicrobial peptides of multicellular organisms. Nature. 2002;415(6870):389-95.

29. Matsuzaki K. Why and how are peptide-lipid interactions utilized for self-defense? Magainins and tachyplesins as archetypes. Biochim Biophys Acta. 1999;1462(1-2):1-10.

30. Mcphee JB, Hancock RE. Function and therapeutic potential of host defence peptides. J Pept Sci. 2005;11(11):677-87.

31. Mookherjee N, Hancock RE. Cationic host defence peptides: innate immune regulatory peptides as a novel approach for treating infections. Cell Mol Life Sci. 2007;64(7-8):922-33. 